\title{
Towards Artifact-free FIB-SEM Datasets: Strategies Before, During, and After Image Acquisition
}

Kedar Narayan $^{1}$, Alexandre Laquerre ${ }^{2}$ and Michael Phaneuf ${ }^{2}$

${ }^{1}$ National Cancer Institute, NIH \& Frederick National Laboratory, Frederick, Maryland, United States, ${ }^{2}$ Fibics Inc, Ottawa, Ontario, Canada

Of the various volume electron microscopy (vEM) approaches used in cell biology, focused ion beam scanning electron microscopy (FIB-SEM) is unique in that the iterative ablation of resin-embedded material is accomplished by an electronically controlled ion beam. In a typical FIB-SEM instrument, two columns oriented at an angle relative to each other are operated in tandem, where energetic Gallium ions are focused and rastered at a glancing angle across a plane of heavy metal stained and resin-embedded cellular material; the newly revealed "cliff face" is then interrogated by a scanning electron beam, resulting in the recording of backscatter or secondary electrons at appropriate detectors [1]. Eschewing mechanical slicing of the specimen sidesteps known artifacts but introduces other challenges. For example, FIB milling can be routinely and consistently generate "slices" in sub-10 nm increments [2,3], however in this regime, the thermal drifts coupled with the sensitivity of the resin to the ion and electron bombardment introduces drifts and warping whose amplitudes approach the slice thickness itself.

In a previous collaboration, we have reported the use of a system of notches in a protective pad deposited above the region of interest - periodic monitoring of notch cross-sections during milling and imaging allows for real time adjustments to the drift measured in the imaging plane, $x y$, as well as in the milling direction, $z[3,4]$. These advances have significantly improved quality of FIB-SEM datasets, but one artifact in particular, colloquially called the "wash effect" or "waterfall effect", remains challenging to eliminate. One hypothesis based on empirical evidence is that as a consequence of the resin reacting to and shrinking locally under an electron beam, areas of the cliff face are no longer milled by the glancing FIB; this exacerbates this local variability effect in the subsequent slice. At some point, the dose imparted from the progressing FIB finally exceeds that required for the ablation of this material and it is rapidly milled away in the direction of the FIB, resulting in a cascading visual effect over several images.

Here we report approaches to alleviate this error, especially in problematic samples: 1. For samples that do not respond well to heavy metallization protocols, addition of simple reagents and an increased curing temperature improves resin resilience to the beam in our hands. 2. During acquisition, improved beam control strategies reduce the appearance of unevenness. Rather than milling away a resin volume corresponding to the targeted slice thickness, say $10 \mathrm{~nm}$, we show that redistributing the image dose from the SEM on a smaller effective volume by acquiring thinner and correspondingly faster slices as thin as 1 $\mathrm{nm}$ causes fewer fluctuations in stability. The thinner and noisier images are then accumulated resulting in slices with the targeted thickness and signal to noise ratio (SNR). Further, slice thickness assignments are now more precise, allowing for more accurate reconstructions. 3. After image stack registration, a simple cut-off or FFT based masking approach along orthogonal planes reduces large contrast variations as the "wash effect" is often cyclical.

Together, by ameliorating a problematic visual artifact generated in FIB-SEM datasets, these approaches further empower FIB-SEM for use by cell biologists who wish to generate accurate nanoscale 3D reconstructions of cellular architectures a variety of specimens.

References 
1. Narayan, K. and Subramaniam, S. (2015). Focused Ion Beams in Biology. Nature Methods Nov;12(11):1021-31

2. Xu, C. S. et al. (2017). Enhanced FIB-SEM systems for large-volume 3D imaging. Elife May 13;6

3. Narayan, K. et al. (2014). Multi-resolution correlative focused ion beam scanning electron microscopy: applications to cell biology. Journal of Structural Biology. Mar 185(3):278-84

4. Phaneuf, M. W. and Lagarec K. G. (2017). Microscopy imaging method and system US9812290B2 filed 31 Jan 2017 \& issued 07 Nov 2017.

We thank Kunio Nagashima and Adam Harned for sample preparation and FIB-SEM image acquisition, respectively. We thank Christian Suloway for help with image processing steps. This project has been funded in whole or in part with Federal funds from the National Cancer Institute, National Institutes of Health, under Contract No. 75N91019D00024. The content of this publication does not necessarily reflect the views or policies of the Department of Health and Human Services, nor does mention of trade names, commercial products, or organizations imply endorsement by the U.S. Government. 